\title{
Prevalencia de problemas éticos en Servicios de Medicina, desde la perspectiva del paciente
}

\author{
Tomás León $\mathrm{R}^{\mathrm{a}}$, Paula Bedregal ${ }^{\mathrm{b}}$, Beatriz Shand $\mathrm{B}^{\mathrm{c}}$. \\ Ethical problems in medical services \\ from the patients' point of view
}

Background: There is a paucity of information on the perception of patients about ethical issues in medical practice. Aim: To determine the type and frequency of ethical problems perceived by patients hospitalized in public and private hospitals. Material and methods: Eighty eight subjects aged 65 years (48\% women), admitted to a public hospital and 44 subjects aged 59 years ( $52 \%$ women) admitted to a private clinic answered a survey about ethical problems. The main issues covered were medical information management, participation in decision making and intimacy of patients. Results: Fifty three percent of patients perceived at least one ethical problem. Patients admitted to the public hospital perceived more communication and intimacy problems than their peers admitted to a private clinic. Conclusions: Approximately half of the interviewed patients acknowledged the presence of ethical problems, especially in the communication and intimacy areas (Rev Méd Chile 2009; 137: 759-65).

(Key words: Confidentiality; Ethics; Physician-patient relations)
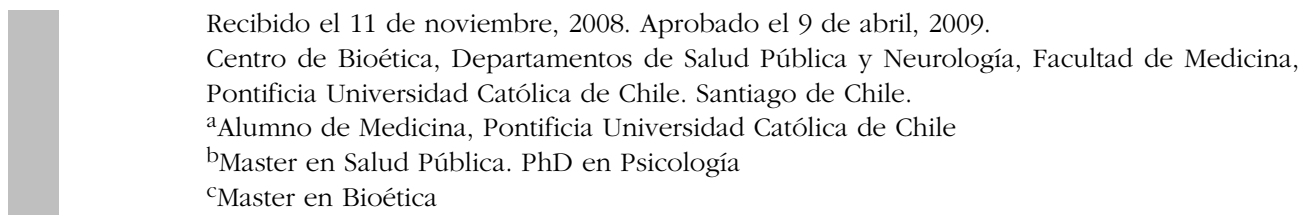

$\mathrm{L}$ a preocupación respecto de la dimensión ética de la práctica de la medicina no es algo nuevo. Pero es en las últimas décadas que se organiza la disciplina de Bioética -término acuñado por Potter en $1971^{1}$ - como el área de la ética que se ocupa de los problemas que se generan en el estudio y manejo de las ciencias relacionadas con los seres vivos y personas.

A pesar de que existe un interés creciente en conceptos como la autonomía del paciente, el

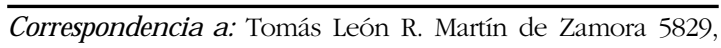
Dpto. 71. Las Condes, Santiago, Chile. Fonos: 5624156429 569 98119292. E mail: tileon@uc.cl consentimiento informado y el conflicto de intereses, la cuantificación empírica de la frecuencia de estos problemas no ha sido suficientemente explorada. Esto puede verse explicado por una serie de problemas metodológicos. El primero de ellos es la dificultad en alcanzar una definición universalmente aceptada y práctica del momento en que un problema médico habitual se convierte en un problema ético. Esta dificultad ha sido reconocida ampliamente en la literatura ${ }^{2}$. Para algunos autores una decisión se torna ética cuando nuestros valores entran en juego en ella, ya que, científicamente, no hay una opción que sobresalga claramente sobre otra ${ }^{2}$. En la literatura científica encontramos algunos esfuerzos por cuantificar la 
presencia de dilemas éticos en distintos escenarios de atención, a través de variados métodos, tales como cuestionarios a los médicos, autorreferencia y decisión en grupo, con o sin la asesoría de un especialista en ética ${ }^{3,4}$. Cabe señalar que la gran mayoría de estos trabajos consideran casi únicamente la opinión del equipo médico tratante para determinar si se ha tenido o no un problema ético en el manejo de un paciente. Según el tradicional trabajo del autor americano Bernard Lo, la frecuencia de problemas éticos detectados por especialistas en bioética, en servicios de medicina, llegaría hasta $30 \%{ }^{4}$.

A pesar de que se han detectado diferencias entre la percepción del equipo médico versus la percepción del paciente, esta última no es un tema ampliamente desarrollado.

En nuestro medio el único trabajo que toma en consideración la opinión de los pacientes en temas éticos es el realizado por Pérez et $\mathrm{al}^{5}$ cuya fecha de publicación data de hace ya 20 años.

Los objetivos de nuestra investigación son:

1. Determinar la frecuencia y tipo de problemas éticos percibidos por pacientes hospitalizados en los servicios de medicina interna de un hospital público y de un hospital privado. Ambos centros clínicos universitarios.

2. Comparar los resultados en los dos ambientes de atención médica.

\section{PACIENTES Y MÉTODO}

Se diseñó un estudio de carácter transversal. La población objetivo fueron todos los pacientes hospitalizados en servicios de medicina interna del Hospital Asistencia Pública de Santiago (HAP) y Hospital Clínico de la Pontificia Universidad Católica de Chile (HCUC). El primero constituye un centro de atención público, especializado en urgencias; el segundo es un hospital universitario de alta complejidad. Ambos ubicados en la capital de Chile, Santiago, en la comuna de Santiago. La muestra correspondió al total de camas de los servicios de medicina de ambos hospitales. Se realizó entre mayo y diciembre de 2007, se obtuvieron 88 pacientes del hospital público y 44 pacientes del hospital privado. Se excluyeron a priori a pacientes con incapacidad de contestar la encuesta debido a un compromiso cualitativo o cuantitativo de conciencia, definido como un Glasgow menor a $15^{6}$. Dos pacientes se negaron a participar, siendo de centros y sexos distintos.

Se diseñó una encuesta para ser autoadministrada (Tabla 1) y que contiene los problemas éticos más frecuentemente señalados en la literatura científica ${ }^{7-11}$ (Tabla 2). Podía ser administrada por el paciente o el encuestador.

Se incluyó un consentimiento informado en el cual se presentó una definición de problema ético basada en la literatura ${ }^{12}$ y que es: "Situaciones en las cuales los pacientes hospitalizados en los servicios de medicina, identifican conflictos en la relación con el médico o con el personal de salud, debido a que la conducta del personal de salud parece inadecuada desde un punto de vista ético. Este conflicto puede deberse, entre otras cosas, a que el paciente se siente pasado a llevar en su dignidad". Se aclaró que la participación era voluntaria y la información confidencial.

Los problemas éticos se delimitaron en cuatro grandes áreas, de acuerdo al reporte de la investigación internacional. Cada una con una o más preguntas:

1. Problemas con el manejo de información:

- La entrega de la información: se investigó si el paciente percibía algún problema con respecto a la cantidad, forma o momento en que era informado.

- El flujo de información: se investigó respecto de cómo perciben los pacientes que se realizó el flujo de información respecto de su enfermedad.

- Consentimiento informado: Se buscó identificar conflictos en relación al proceso y firma del consentimiento informado.

2. Problemas de participación en el tratamiento:

- Se consultó qué problemas éticos reconoce el paciente en el proceso de toma de decisiones con respecto a su tratamiento.

3. Conflictos de intereses en el acto médico:

- Nuestro interés fue reconocer en qué medida el paciente detecta en su relación con el personal de salud estos conflictos de intereses y los reconoce como problemas éticos.

4. Problemas éticos relacionados con el respeto por la intimidad del paciente:

- Quisimos averiguar en qué medida el paciente se siente afectado en su pudor y privacidad. 
Tabla 1. Resumen de la encuesta

\begin{tabular}{|c|c|c|c|}
\hline Pregunta & $\mathrm{Si}$ & No & Especifique \\
\hline $\begin{array}{l}\text { Respecto de la información relacionada a su problema de salud: } \\
\text { ¿Identificó un conflicto en la CANTIDAD de información que recibió? } \\
\text { ¿Identificó un conflicto en LA FORMA (o el modo) en que se entregó } \\
\text { la información? } \\
\text { ¿Identificó un algún conflicto respecto de A QUIÉN se le entregó la } \\
\text { información? } \\
\text { ¿Ha sido sometido a alguna intervención o procedimiento? } \\
\text { Si su respuesta es SI responda la siguiente pregunta: } \\
\text { ¿Se le solicitó a Ud. (o a su representante) firmar un papel referente a } \\
\text { su aprobación con respecto a dicha intervención o procedimiento } \\
\text { (consentimiento informado)? } \\
\text { Si su respuesta es SI responda la siguiente pregunta: } \\
\text { ¿Identificó un conflicto en relación a la forma en que se firmó el } \\
\text { consentimiento informado, por ejemplo que no se le explicara de manera } \\
\text { adecuada? } \\
\text { ¿Identificó un conflicto en relación a su participación en decisiones con } \\
\text { respecto a su enfermedad, tratamientos o procedimientos a seguir? } \\
\text { ¿Cree ud. que SU SALUD fue lo más importante para el equipo médico } \\
\text { al momento de tomar decisiones? } \\
\text { ¿Cree que los integrantes del equipo médico han hecho todo lo } \\
\text { posible por proteger su intimidad al momento de examinarlo(a)? } \\
\text { ¿Ha existido alguna situación en que procedimientos realizados a } \\
\text { usted o a otro paciente le hayan hecho sentir incómodo(a) y/o que se } \\
\text { ha pasado a llevar su privacidad? } \\
\text { ¿Ha percibido usted, durante su hospitalización, algún otro problema } \\
\text { ético, que hasta ahora no se haya mencionado? }\end{array}$ & & & \\
\hline
\end{tabular}

Tabla 2. Problemas éticos más frecuentemente reportados

\begin{tabular}{ll}
\hline Trabajo & Principales problemas descritos \\
\hline Lo B, et al. Frequency of ethical dilemmas & 1. Mantención de tratamiento y \\
in a medical inpatient service, 1981 & consentimiento informado \\
& 2. Entrega de información \\
& 3. Relaciones entre médicos \\
Perez, et al. Relación médico-paciente: & 4. Recursos limitados \\
Estudio comparativo entre dos servicios & 1. Nivel de información \\
de Medicina Interna. 1988 & 2. Tiempo de atención \\
Hurst, S, A, Hull, S, C, DuVal, G, Danis, M. & 3. Uso del tiempo de atención \\
How physicians face ethical difficulties: & 1. Paciente incurable \\
a qualitative analysis, 2005 & 2. Situaciones con otros médicos \\
& 3. Autodeterminación del paciente \\
& 4. Proporcionalidad de tratamiento
\end{tabular}


Adicionalmente la encuesta preguntó por sugerencia de temas que no se hubieren cubierto con las preguntas anteriores. La encuesta fue revisada por un grupo de académicos y estudiantes de medicina, para detectar áreas temáticas que pudieran ser omitidas, confusas o corregir la redacción.

Luego se aplicó a un grupo piloto de 16 pacientes, quienes al terminarla opinaron sobre su dificultad y determinaron preguntas poco claras. La versión definitiva de la encuesta está estructurada en áreas temáticas que incorporan una a dos preguntas, que se contestaban sí o no. La encuesta se aplicó en los dos servicios de medicina, por tres alumnos de cuarto año de medicina, previamente entrenados y que no participaban del cuidado del paciente.

Se realizó un análisis estadístico descriptivo de las variables de interés y se compararon los resultados entre ambos ambientes de práctica médica mediante pruebas de diferencias de proporciones y de medias. Las respuestas positivas fueron catalogadas como de valor 2, y 1 aquellas negativas. Se estableció un puntaje total de la encuesta de 16 puntos. La escala presentó una consistencia interna de 0,64 (Kuder-Richardson 20), lo que es aceptable para un estudio exploratorio. El puntaje máximo observado fue de 16. Finalmente se realizó un análisis de regresión múltiple de carácter explicativo, cuya variable independiente fue el puntaje de la escala de problemas éticos para determinar cuál variable explica el puntaje observado, y varios análisis de regresión logística considerando como variables dependientes: reportar al menos un problema ético y la respuesta a cada ítem.
Este proyecto cumple con las normas éticas exigidas internacionalmente y fue aprobado por el Comité de Ética de Investigación del Centro de Investigaciones Médicas de la Pontificia Universidad Católica de Chile. La aplicación de la encuesta contó con la autorización de los jefes de ambos servicios.

\section{Resultados}

Las características de la muestra alcanzada según lugar de hospitalización se señalan en la Tabla 3. Al comparar ambos grupos, se observó que el promedio de edad y el tiempo de estadía es menor en el HCUC ( $\mathrm{p}<0,05)$, y que el nivel de educación es menor en el HAP $(p<0,05)$. En promedio, $53 \%$ de pacientes refirió al menos un tipo de problema ético. En el HCUC este porcentaje fue de $45,5 \%$ y en el HAP de 56,8\% (p =0,147). El número promedio de problemas percibidos en el HCUC fue de 0,63 y en el HAP fue de 1,27 ( $\mathrm{p}=0,015)$.

La tabla siguiente (Tabla 4) muestra el comportamiento de la muestra con relación a la percepción de problemas éticos. Con relación al área sobre "Manejo de la Información", aproximadamente $12 \%$ identifica algún problema. Dentro de las quejas que se refieren a falta de información, se dijo: "Quedé con dudas, es poca información".

Sobre la forma algunos señalan que: "Fue muy chocante". Finalmente, sobre a quién se le entrega la información se señala: "Se me debió preguntar a quién se le debía dar la información y no a todo el mundo que le preguntaba".

Si bien es cierto no se encontró diferencias estadísticamente significativas entre ambos esta-

Tabla 3. Composición de la muestra

\begin{tabular}{|llllc|}
\hline Variable & H AP & HCUC & Total & Valor $\mathbf{p}$ \\
\hline Edad (años) & 64,6 & 58,8 & 60 & $<0,0001$ \\
Días hospitalización & 8,1 & 5,0 & 7,1 & 0,011 \\
Mujeres (\%) & 47,7 & 61,4 & 52,3 & 0,098 \\
Antecedente enfermedad crónica (\%) & 67,0 & 68,2 & 67,4 & 0,529 \\
Hospitalización previa (\%) & 68,2 & 77,3 & 71,2 & 0,189 \\
Nivel educacional (\%) & Básica: 61,4 & Básica: 22,8 & Básica: 49,0 & 0,0001 \\
& Universitario: 6,8 & Universitario: 38,6 & Universitaria: 17,4 & \\
\hline
\end{tabular}


Tabla 4. Frecuencia relativa de problemas éticos detectados según establecimiento

\begin{tabular}{|lrrrr|}
\hline Problema ético & $\begin{array}{r}\text { H AP } \\
\text { (\% total) }\end{array}$ & $\begin{array}{r}\text { H C C } \\
\text { (\% total) }\end{array}$ & $\begin{array}{r}\text { Total } \\
\text { (\% total) }\end{array}$ & Valor $\mathbf{p}$ \\
\hline Cantidad de información entregada & 15,9 & 2,3 & 11,4 & 0,015 \\
La forma de entregar la información & 12,5 & 11,4 & 12,1 & 0,547 \\
A quién se le entregó la información médica & 8,0 & 4,5 & 6,8 & 0,371 \\
No recuerdan haber firmado consentimiento & & & & \\
informado para procedimientos & 44,2 & 17,2 & 33,3 & 0,015 \\
Aplicación del consentimiento informado & 8,7 & 8,3 & 8,5 & 0,679 \\
Participación en la toma de decisiones & 25,0 & 4,5 & 18,2 & 0,002 \\
Interés primario del médico no está & & & & \\
en la salud del paciente & 6,8 & 0 & 4,5 & 0,083 \\
Falta de intimidad & 20,5 & 9,1 & 16,7 & 0,077 \\
Problema ético en el respeto a la intimidad & & & & \\
de otro paciente que le hizo sentirse incómodo & 20,5 & 2,3 & 14,4 & 0,003 \\
\hline
\end{tabular}

blecimientos con relación a los conflictos con respecto a quién que se le entrega la información, este aspecto fue el único que mostró diferencias significativas por sexo ( $\mathrm{p}=0,011$ ), ya que fue señalado con mayor frecuencia por hombres $(12,7 \%)$ que mujeres $(1,4 \%)$. El área sobre el uso y aplicación del consentimiento informado fue la más problemática, ya que 33\% señalaron no haber firmado consentimiento. De las que firmaron, algunos tuvieron problemas con la aplicación y señalaron que: "No lo leí", "no sabía lo que era el examen".

Los problemas con la participación en la toma de decisiones constituyen el segundo problema más frecuente. Un paciente mencionó: "No se tomó en cuenta mi opinión frente a mi operación". El área de conflicto de interés fue la menos percibida por los pacientes. Los únicos que percibieron problemas fueron pacientes del hospital público. Las razones para responder afirmativamente esta pregunta fueron, entre otras: "Sentí que era como un trámite".

Con relación al trato recibido, se hacen referencias tanto personales como a otros pacientes. En este último aspecto algunos pacientes señalaron: "Cuando maltratan a la gente que no puede defenderse", "trato brusco". Finalmente en el espa- cio abierto para otros problemas percibidos 19\% de los pacientes señaló haber percibido otro problema que no había sido reconocido en ninguna de las preguntas anteriores. Cabe mencionar que no se encontró un problema definido en esta pregunta. La regresión múltiple mediante técnica de pasos sucesivos, muestra que sólo dos variables explican el comportamiento de la varianza en el puntaje del cuestionario: el lugar de hospitalización $(0=\mathrm{HAP})$ y el nivel educacional $(0$ =educación básica completa o incompleta $)\left(R^{2}\right.$ $=0,092$ ), ambas variables con alta colinearidad, siendo la más importante el lugar de hospitalización. El coeficiente de regresión beta estandarizado para el lugar de hospitalización es de 0,312, y el del nivel de educación de -0,221. Al establecer como medida de resultado al menos un problema detectado, mediante técnica de regresión logística, se obtiene que la única variable que ingresa al modelo de manera estadísticamente significativa ( $\mathrm{p}=0,006)$ es el lugar de hospitalización con un OR de 8,3 [1,1 - 64,9].

Finalmente, 5 items cuentan con alguna variable de las recolectadas que se asocia de manera significativa a contestar de manera afirmativa. La Tabla 5 muestra los OR de aquellas variables que explican el comportamiento de cada ítem del cuestionario. 
Tabla 5. Asociaciones significativas entre ítems y variables en estudio

\begin{tabular}{|c|c|c|c|}
\hline Ítem & Variable asociada & OR (IC 95\%) & Valor $p$ \\
\hline $\begin{array}{l}\text { Cantidad de información } \\
\text { entregada }\end{array}$ & $\begin{array}{l}\text { Lugar de hospitalización } \\
(\text { HCUC }=0)\end{array}$ & $8,25[1,1-64,9]$ & 0,045 \\
\hline $\begin{array}{l}\text { A quién se le entrega } \\
\text { la información médica }\end{array}$ & $\begin{array}{l}\text { Sexo } \\
(\text { Masculino }=0)\end{array}$ & $0,1[0,01-0,82]$ & 0,032 \\
\hline $\begin{array}{l}\text { Participación en la } \\
\text { toma de decisiones }\end{array}$ & $\begin{array}{l}\text { Lugar de hospitalización } \\
(\mathrm{HCUC}=0)\end{array}$ & $7,1[1,6-31,8]$ & 0,01 \\
\hline \multirow[t]{2}{*}{$\begin{array}{l}\text { Problemas con la intimidad } \\
\text { en otros pacientes }\end{array}$} & $\begin{array}{l}\text { Lugar de hospitalización } \\
(\mathrm{HCUC}=0)\end{array}$ & $18,2[2,2-14,8]$ & 0,007 \\
\hline & Educación (>Básica completa =0) & $0,29[0,1-0,84]$ & 0,023 \\
\hline
\end{tabular}

DiSCUSIÓN

Nuestro estudio muestra una muy alta prevalencia de problemas éticos desde la perspectiva del paciente. Es posible plantear, sin embargo, que por las limitaciones de nuestro método de pesquisa de los problemas, un grupo importante de problemas éticos presentes en estos servicios, no fueron incluidos. Tal como lo muestran estudios enfocados en la percepción del médico, los problemas relacionados a toma de decisiones en enfermos terminales o no competentes, se cuentan entre los problemas más frecuentes de un servicio de medicina interna ${ }^{13}$. En nuestro estudio mostramos que alrededor de la mitad de los pacientes competentes hospitalizados reconocen uno o más problemas éticos, sin embargo, al incluir casos de pacientes no competentes, esta proporción podría ser mucho mayor.

Es importante destacar que no hubo diferencias significativas entre los dos centros en cuanto al porcentaje de pacientes que perciben uno o más problemas, sin embargo, el patrón de respuesta es claramente distinto y las diferencias no se explican por edad, sexo o tiempo de estadía.

Esta diferencia podría deberse, en primer lugar, a una distinta sensibilidad a los problemas. Esto se ha descrito, por ejemplo, en grupos de médicos de distinta raza ${ }^{14}$. La mayor sensibilidad por parte de los pacientes podría explicarse por factores no evaluados por la encuesta, como distintos condicionantes sociales o nivel socioeconómico. Es difícil plantear sin embargo, que un mayor nivel so- cioeconómico se asocie a una menor sensibilidad frente a los problemas. Sobre todo si vemos que la mayor escolaridad de los pacientes en nuestro estudio se asoció a mayor presencia de problemas en la pregunta referente al respeto a la intimidad, lo que permite suponer que exista una asociación en sentido opuesto. Por lo tanto, la diferencia socioeconómica podría ocultar una diferencia aún mayor que la detectada.

Planteamos entonces que la mayor percepción de problemas éticos reconocidos en el hospital público se debe a la existencia real de esta diferencia. Una primera explicación para la misma tiene relación con las condiciones de trabajo disímiles (tiempo dedicado a cada paciente, espacio físico, continuidad de los cuidados por un mismo equipo). Es posible también, que el estilo de relación médico-paciente o entre el paciente y el personal de salud, en uno u otro servicio, sea distinto.

Al concentrarnos en los problemas que fueron significativamente más frecuentes en el hospital público, proponemos el siguiente análisis: Dos de ellos tienen como denominador común la comunicación en la relación médico-paciente: participación en la toma de decisiones y manejo de la información. El tercero corresponde al manejo de la intimidad. En éste último llama la atención que no encontramos diferencias significativas por sexos en la percepción de este problema.

Por lo tanto, nuestro estudio muestra que para los pacientes en el servicio público las dificultades en la comunicación con el equipo de salud son la 
causa primaria de gran parte de los problemas éticos que perciben. Esto podría explicarse, nuevamente, por condiciones de trabajo disímiles. Tal como lo mostró el estudio descriptivo de Pérez et $\mathrm{al}^{5}$, existe una real diferencia de tiempo dedicado por paciente en el sistema público y privado. Una segunda explicación, es que el modelo de atención en uno u otro escenario sea distinto. Un trabajo reciente realizado en un hospital público de Brasil ${ }^{15}$, mostró la percepción de una clara asimetría en la relación médico-paciente. Nuestro estudio sugiere, en esta misma línea, que un patrón paternalista de manejo de los pacientes no parece el más adecuado en ningún escenario y afirma la necesidad de erradicar una mal entendida subsidiariedad en la toma de decisiones.

Es importante mencionar en este punto, que el modelo de atención participativo, no sólo parece más idóneo y satisfactorio desde la perspectiva del paciente, sino que también ha demostrado favorecer el autocuidado y la adherencia del paciente al tratamiento ${ }^{14}$.

\section{REFERENCIAS}

1. Potter, Van Rensselaer. Bioethics: Bridge to the Future. New Jersey, Estados Unidos: Editorial Englewood Cliffs: Prentice-Hall. 1971; 123.

2. Braunack-Mayer, Annette, Joy. What makes a problem an ethical problem? An empirical perspective on the nature of ethical problems in general practice. J Med Ethics 2001; 27: 98-103.

3. Ruhnke GW, Wilson SR, Akamatsu T, Kinoue T, TAKshima Y, Golstein MK et al. Ethical decision making and patient autonomy: a comparison of physicians and patients in Japan and the United States. Chest 2000; 118: 1172-82.

4. Lo B, Schroeder SA. Frequency of ethical dilemmas in a medical inpatient service. Arch Intern Med 1981; 141: 1062-4.

5. Pérez C, Jalil R, Canals C, Soto S, Pinto Ji, Bedregal P, Godoy D. Relación médico-paciente: estudio comparativo entre dos servicios de medicina interna. Rev Méd Chile 1998; 116: 1239-45.

6. Teasdale G, Jennett B. Assessment of coma and impaired consciousness. A practical scale. Lancet 1974; 2: 81-4.

7. SimON A. Support for ethical dilemmas in individual cases: experiences from the Neu-Mariahilf Hospital in Goettingen. J Med Ethics 2001; 27: 18-20.
Según nuestros resultados en el único escenario en el cual el médico debería adoptar un rol protector del paciente, se refiere al respeto de la intimidad en personas de baja escolaridad (que podrían reconocer menos este tipo de problemas).

Concluimos reflexionando que este estudio es una más de las pruebas que validan la necesidad de implementar una formación ética profunda del equipo de salud, orientada a aumentar la capacidad de los integrantes de este equipo para pesquisar y manejar problemas éticos ${ }^{4}$ y el fortalecimiento de las habilidades de comunicación efectiva.

\section{Agradecimientos}

Al Departamento de Bioética de la Pontifica Universidad Católica y en especial a la Dra. Lorena Mosso por su constante apoyo e inspiración. A los ayudantes alumnos que nos apoyaron en la aplicación de la encuesta. A nuestras familias por el apoyo constante.

8. Pérez-Cárceles MD, Pereñíguez-Barranco Je, OsunaCarrillo de Albornoz E, luna-Maldonado A. Derecho de información de los pacientes: influencia de las características socioprofesionales en atención primaria. Atención Primaria 2006; 2: 69-74.

9. Tremayne-Lloyd T. Confidentiality. Disclosure to a patient's family. Can Fam Physician 2003; 49: 300-1.

10. Pérez-Cárceles MD, Pereñíguez Je, Osuna E, Luna A. Balancing confidentiality and the information provided to families of patients in primary care. J Med Ethics 2005; 31: 531-5.

11. Clark P. Confidentiality and the physician-patient relationship-ethical reflection from a surgiral waiting room. Med Sci Monit 2002; 8: SR31-4.

12. Gómez-Lobo A. Los bienes humanos, ética de la ley natural. Santiago, Chile: Editorial Mediterráneo Ltda. 2006; 241.

13. Duval G, Clarridge B, Gensler G, Danis M. A National Survey of U.S. Internists' Experiences with Ethical Dilemmas and Ethics Consultation. J Gen Intern Med 2004; 19: 251-8.

14. Fuertes JN, Mislowack A, Bennett J, Paul L, Gilbert TC, FONTAN G, BOYlan LS. The physical- patient working alliance. Patient Educ Couns 2007; 66: 29-36.

15. Albuquerque MC, Roffé R. The asymmetrical relationship between the health care professional and the patient. J Int Bioethique 2008; 19: 165-79, 205-6. 